\title{
Recurrent pancreatic cancer patient treated by chemotherapy and focused ultrasound surgery. A case report
}

\author{
Dobromir Dimitrov ${ }^{1,2}$, Nadya Stanislavova ${ }^{2}$, Tsanko Yotsov ${ }^{1}$, Kun Zhou ${ }^{3}$ \\ ${ }^{1}$ Department of Surgical Propaedeutics, Medical University-Pleven, Bulgaria, ${ }^{2} \mathrm{HIFU}$ Center, St. Marina Hospital, \\ Medical University-Pleven, Bulgaria, ${ }^{3}$ Clinical Center for Tumor Therapy of the Second Affiliated Hospital of Chong- \\ qing Medical University, Chongqing, China
}

\begin{abstract}
We present a case of recurrent pancreatic cancer diagnosed by computer tomography (CT) and positron emission tomography (PET), 7 months after Whipple radical surgery in a 61-year-old female patient. The patient was successfully treated by focused ultrasound surgery (FUS) by innovative high intensity focused ultrasound device. The patient had no complications. Multiple cycles of chemotherapy were done. Twelve months after FUS the new PET-CT showed no evidence of metabolite active zone in the area of ablation and no progression of disease. The presented case is unique according to the literature as a local recurrence after radical surgery for pancreatic cancer, successfully managed by local FUS ablation and adjuvant chemotherapy.

Keywords: high intensity focused ultrasound ablation; pancreatic cancer; chemotherapy
\end{abstract}

\section{Introduction}

Radical surgery for pancreatic cancer has median survival for all stages of 12.6 months. It is however possible in only $20 \%$ of the patients [1,2]. Despite the $\mathrm{R} 0$ resection, most patients will experience a local recurrence or progression of disease with hepatic metastases or peritoneal carcinomatosis [3]. Recurrences remain a challenge for treatment as operative treatment and chemoradiation have limited efficiency and the advancement of stage of the disease. The pessimistic attitude of clinicians towards

Received 19.05.2019 Accepted 24.07.2019

Med Ultrason

2020, Vol. 22, No 2, 247-249

Corresponding author: Dobromir Dimitrov

Medical University-Pleven

1-st KlimentOhridski street Pleven,

PC 5800, Bulgaria

E-mail: dobri_dimitrov@abv.bg recurrent pancreatic cancer is very common, which leads to untreated recurrence or palliation of the patients [4].

The present report of a patient with recurrent pancreatic cancer after radical surgery treated successfully with chemotherapy and local ablation with focused ultrasound surgery (FUS).

\section{Case report}

In 2014 a 61-year old patient underwent a successful Whipple's procedure for adenocarcinoma of the head of the pancreas. The stage of the disease was T2N0(10) M0, I stage. Seven months after the operation because of abdominal and lower back pain, a contrast enhanced computer tomography (CT) was performed. The CT evidenced a tumor in the body of the pancreas around metal clips suspicious of local recurrence. A positron emission tomography CT (PET-CT) was performed for confirmation of the diagnosis. It shows a tumor formation at the 
site of operation with dimensions $24 / 20 / 22 \mathrm{~mm}$, volume $5.5 \mathrm{ml}$ and max SUV 7.4 (fig 1). The tumor mass was in contact with the inferior cava vein, superior mesenteric artery and aorta. The case was presented for discussion at the High Intensity Focused Ultrasound (HIFU) center in University Hospital "St. Marina", MU-Pleven. At this point the patient reported pain measured by the visual analog scale (VAS) at 8 out of 10 points, which was treated with $50 \mathrm{mg}$ tramadol two times a day. The comorbidity of the patient was diabetes mellitus and hypothyroidism. The European Organisation for Research and Treatment of Cancer (EORTC) instrument Quality of Life Questionnaire cancer 30 was used to evaluate the patient before and after ablation. The baseline answers of the patient showed poor global health status (GHS) with 0 points and moderate pancreatic pain (50 points) treated with drugs. FUS with HIFU and adjuvant treatment with Gemcitabine $\left(1,600 \mathrm{mg} / \mathrm{m}^{2}\right.$ i.v. weekly) were proposed to the patient.

After signing an informed consent form, the FUS treatment was performed. The patient was prepared for general anesthesia beforehand with no out of norm tests and consultation examinations. The patient was positioned prone on the HIFU table model JC Chongqing Haifu Medical Technology Co. Ltd., China. Between the anterior abdominal wall of the patient and the HIFU transducer was placed a special balloon used to compress the viscera, shorten the acoustic pathway and cool the skin during procedure. The FUS was performed with duration 105 minutes and mean dose per $1 \mathrm{ml}$ of tumor mass $12307.30 \mathrm{~J} / \mathrm{ml}$. First hyperechogenic changes in real time in the pancreas appeared at 337 seconds as an ultrasound sign of coagulative necrosis (fig 2). The patient was discharged after 5 days in a good general condition, without complications. Adjuvant chemotherapy was performed with 10 cycles of Gemcitabine after FUS. The control CT showed wide hypodense zone, with no sign of enhanced contrast area (fig 3). At the same time VAS was reduced to 3 out of 10 and the patient ceased to use Tramadol. Us-

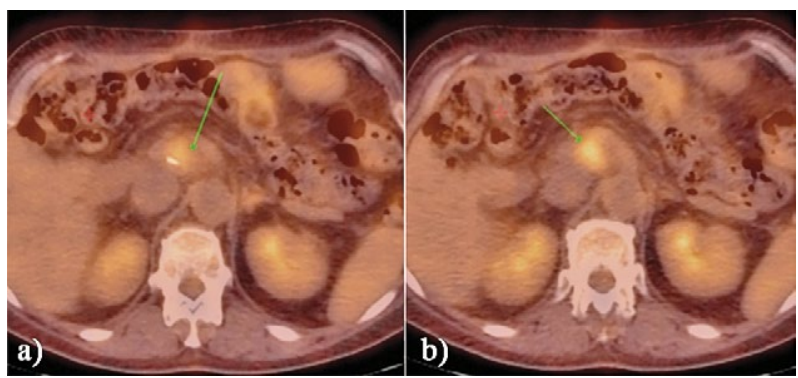

Fig 1. PET-CT data of metabolic active zone SUV max 7.4 in the postoperative area around the metal clips (A) and in the residual tissue of the pancreas $(\mathrm{B})$

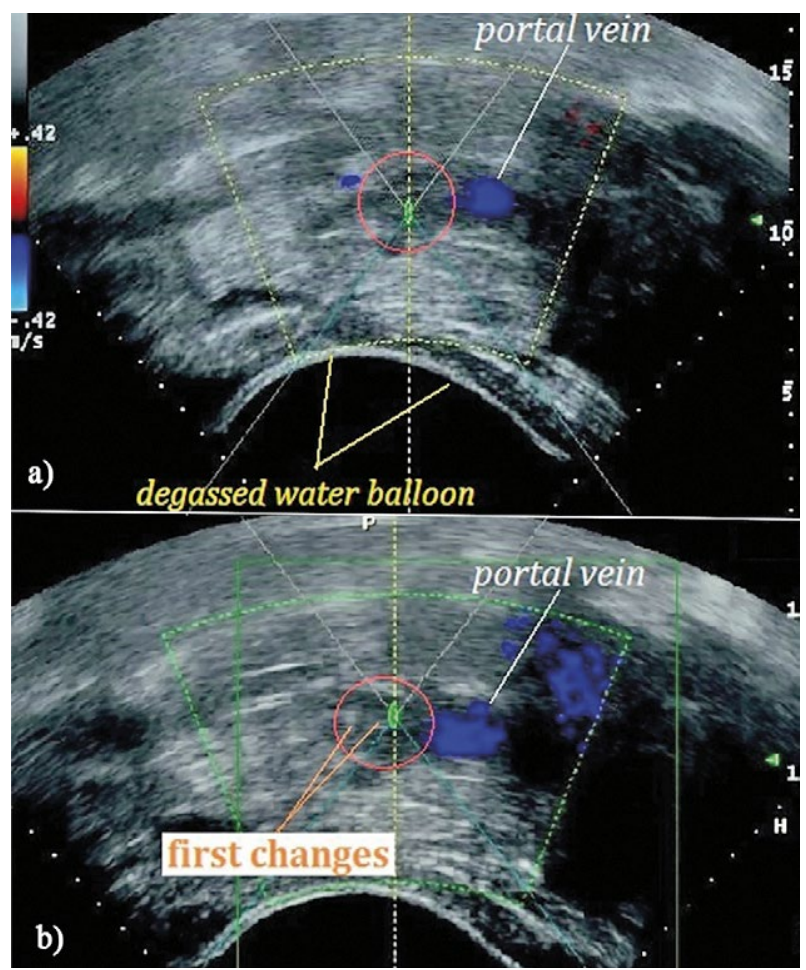

Fig 2. Image from the HIFU device software showed some parameters of the ablation (A) and first hyperechogenic changes during FUS of the recurrent cancer (B).

ing the EORTC standards GHS reached 67 points and the pancreatic pain was 0 according to the patient. Twelve months after FUS, PET-CT showed no progression of the disease (fig 4). The registered survival of the patient after FUS was 24 months and 32 months after diagnosis.

\section{Discussions}

FUS using a HIFU as a non-invasive procedure that can be used in treatment of benign or malign tumors and can be guided with no need of puncture, needle or incision of the skin surface. The method uses HIFU waves, focused on a lesion with the help of special equipment with build in imaging device - MRI or ultrasound, and calculation devices for precise focus of the ultrasound waves. When the ultrasound waves reach the target locus they superpose and the result wave has higher energy then the summer energy of the separate waves. It is absolutely non-invasive for the surround tissues and the skin. The result wave causes rapid increase in the temperature of the tumor mass and cavitation. There have been trials with European and Asian patients that show better therapeutic results in advanced pancreatic cancer $[5,6]$.

Median time to early recurrence is 3.7 months and median overall survival with chemotherapy is 9.3 months 


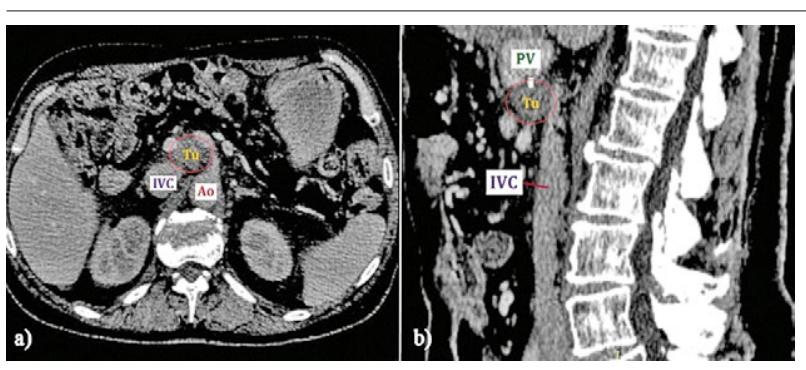

Fig 3. Control CT in 1 month after FUS showed a hypodense area around the metal clips after surgery and abdominal vessels in axial plan (A) and in a front of the inferior cava vein in sagittal plan (B). IVC - Inferior vena cava, Ao - Aorta, PV - portal vein, $\mathrm{Tu}$ - tumor

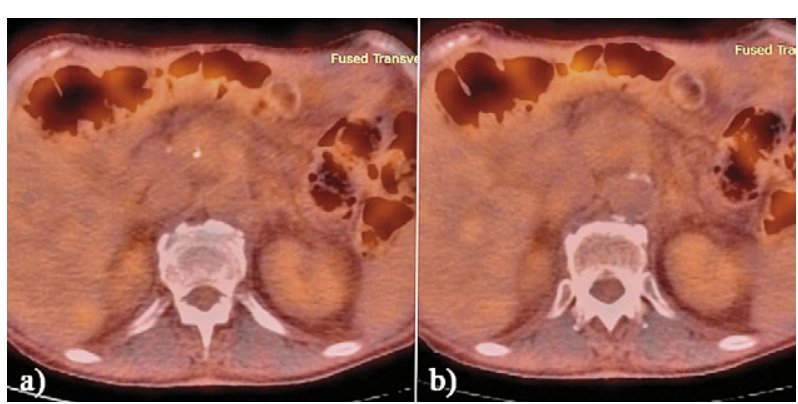

Fig 4. PET-CT data evidenced no metabolic active zone in the area of ablation and no progression of disease 1 year after FUS $(\mathrm{A}, \mathrm{B})$

[7]. The recurrence in our case was diagnosed 7 months after Whipple resection. Resection of recurrent pancreatic cancer is uncommon and performed only in selected cases $[3,4]$. In the presented case the pancreatic surgeons did not offer a resection of the recurrence. According to the MDT FUS was performed in one go with a registered very good result, no complications and necrosis confirmed by CT. In the literature there are few cases successfully treated by HIFU and chemotherapy for locally advanced pancreatic cancer and no recurrent cases $[8,9]$. Wilkowski et al. reported median overall survival in patients with recurrence after pancreatic resection treated with chemoradiation of 17.5 months and median survival after initial diagnosis 27.2 months [10].
The presented case is unique according to the literature as a local recurrence after radical surgery for pancreatic cancer, successfully managed by local FUS ablation and adjuvant chemotherapy.

\section{References:}

1. Hsueh CT. Pancreatic cancer: current standards, research updates and future directions. J Gastrointest Oncol 2011;2:123-125.

2. Bilimoria KY, Bentrem DJ, Ko CY, et al. Validation of the 6th edition AJCC Pancreatic Cancer Staging System: report from the National Cancer Database. Cancer 2007;110:738744.

3. Miyazaki M, Yoshitomi H, Shimizu H, et al. Repeat pancreatectomy for pancreatic ductal cancer recurrence in the remnant pancreas after initial pancreatectomy: is it worthwhile? Surgery 2014;155:58-66.

4. Sperti C, Moletta L, Merigliano S. Multimodality treatment of recurrent pancreatic cancer: Myth or reality? World J Gastrointest Oncol 2015;7:375-382.

5. Marinova M, Rauch M, Mücke M, et al. High-intensity focused ultrasound (HIFU) for pancreatic carcinoma: evaluation of feasibility, reduction of tumour volume and pain intensity. Eur Radiol 2016;26:4047-4056.

6 . Wu F. High intensity focused ultrasound: a noninvasive therapy for locally advanced pancreatic cancer. World J Gastroenterol 2014;20:16480-16488.

7. Fischer R, Breidert M, Keck T, Makowiec F, Lohrmann C, Harder J. Early recurrence of pancreatic cancer after resection and during adjuvant chemotherapy. Saudi J Gastroenterol 2012;18:118-121.

8. Yuan Y, Shen H, Hu XY, Gu FY, Li MD, Zhong X. Multidisciplinary treatment with chemotherapy, targeted drug,and high-intensity focused ultrasound in advanced pancreatic carcinoma. Med Oncol 2012;29:957-961.

9. Lau PC, Zheng SF, Ng WT, Yu SC. Inoperable pancreatic adenocarcinoma rendered completeremission by high-intensity focused ultrasound concurrentwith gemcitabinecapecitabine chemotherapy: case report and topic review. J Dig Dis 2012;13:60-64.

10. Wilkowski R, Thoma M, Bruns C, Dühmke E, Heinemann V. Combined chemoradiotherapy for isolated local recurrence after primary resection of pancreatic cancer. JOP 2006; 7:34-40. 